\title{
Update on Bone-Conduction Auditory Brainstem Responses: A Review
}

\author{
Young Joon Seo ${ }^{1}$, Chanbeom Kwak ${ }^{2}$, Saea Kim², Yoon Ah Park', \\ Kyoung Ho Park ${ }^{3}$, and Woojae Han ${ }^{4}$ \\ ${ }^{1}$ Department of Otorhinolaryngology, Yonsei University Wonju College of Medicine, Wonju, \\ ${ }^{2}$ Department of Speech Pathology and Audiology, Graduate School, Hallym University, Chuncheon, \\ ${ }^{3}$ Department of Otolaryngology-Head and Neck Surgery, The Catholic University of Korea College of Medicine, Seoul, \\ ${ }^{4}$ Division of Speech Pathology and Audiology, Research Institute of Audiology and Speech Pathology, College of Natural Sciences, \\ Hallym University, Chuncheon, Korea
}

Received December 5, 2017

Revised December 20, 2017

Accepted December 20, 2017

\author{
Address for correspondence \\ Woojae $\mathrm{Han}, \mathrm{PhD}$ \\ Division of Speech Pathology and \\ Audiology, College of Natural Sciences, \\ Hallym University, \\ 1 Hallymdaehak-gil, \\ Chuncheon 24252, Korea \\ Tel +82-33-248-2216 \\ Fax +82-33-256-3420 \\ E-mail_woojaehan@hallym.ac.kr
}

Auditory brainstem responses (ABR) have been used as a powerful and the most common objective tool to evaluate hearing sensitivity and to diagnose the types of hearing loss and neurological disorders, through the auditory peripheral pathway to a central level of the brainstem, since 1971. Although bone-conduction (BC) ABR could be an alternative to air-conduction (AC) ABR, as the former overcomes some limitations of the latter, the majority of clinicians rarely utilize it due to a lack of knowledge and no routine test administration. This review presents the weaknesses of $\mathrm{AC} A B R$ that apply to all clinical population, and discusses the development of BC ABR. The optimal placements of bone oscillators to obtain favorable clinical outcomes in infants, children, and adults, and the appropriate stimuli for BC ABR are examined. While providing absolute thresholds and latencies of $B C A B R$ based on previous studies compared to $A C A B R$, this review includes clinical data of infants and young children with both normal hearing in terms of maturation, and with pathology such as congenital external auditory canal atresia. We recommend the future clinical application of BC ABR for candidacy as well as for patients with $\mathrm{BC}$ hearing implants.

J Audiol Otol 2018;22(2):53-58

KEY WORDS: Bone-conduction auditory brainstem response · Bone oscillator - Atresia.

\section{Introduction}

It is well acknowledged that the auditory brainstem response (ABR) test is regarded as an objective test through the auditory peripheral pathway to a central level of the brainstem, and usually evaluates the hearing sensitivity and diagnoses the types of hearing loss and neurological disorders $[1,2]$. Many clinicians have used ABR of especially air-conduction (AC) for patients who might suspect those pathologies. As a result, AC ABR test has been a useful for obtaining clinical outcomes while also providing absolute values of la-

This is an Open Access article distributed under the terms of the Creative Commons Attribution Non-Commercial License (http://creativecommons.org/licenses/by-nc/4.0/) which permits unrestricted non-commercial use, distribution, and reproduction in any medium, provided the original work is properly cited. tency and amplitude from ABR waves.

Nevertheless, there are some limitations to AC ABR in the following specific situations: 1) patients who have multiple disorders that could be attributed to cognitive ability or behavioral self-control [3], 2) populations that have conductive hearing loss such as external auditory canal atresia (EACA), aural atresia, and microtia [4]. The most common cause of failing to obtain AC ABR thresholds in infants is due to conductive deafness [5]. In other words, children and adults who could check behavioral hearing thresholds through both airand bone-conduction pathways might be conducted by only $\mathrm{AC}$ ABR in general and also assumed any possible conductive problem. However, this is not a simple approach for infants who have undergone diagnostic ABR testing, especially for those who have failed multiple neonatal hearing screening tests that have only examined the AC pathway (i.e., from the 
outer ear to the brainstem). Newborns in the intensive care nursery are at increased risk for otitis media [6,7]. The incidence of otitis media with effusion in this population has been reported to be as high as 57 to $67 \%$. Thus, diagnostic ABR protocols need to determine whether there is an important conducting element [6]. We suggest that bone-conduction (BC) ABR could be an alternative to overcome these limitations. The previous studies have displayed that the stimuli pathway in $\mathrm{AC}$ and $\mathrm{BC} \mathrm{ABR}$ may show different characteristics. That is, $\mathrm{AC} \mathrm{ABR}$ presents the stimuli through the ear canal using headphones or inserted earphones, whereas BC ABR uses bone vibrators that directly simulate the cochlea of the inner ear without passing through the outer and middle ear. Thus, the bone vibrator presents the stimuli to the temporal bone so that vibrations could be transmitted directly into the cochlea [8]. This information is crucial for the comparison between $\mathrm{AC}$ and $\mathrm{BC} \mathrm{ABR}$ and is needed to determine the subsequent steps for appropriate follow up and parent counseling. Hall had recommend $\mathrm{BC}$ ABR testing in that it could reduce unnecessary surgeries and screen patients who have low reliability for AC testing due to outer or middle ear dysfunction [9]. Although there are several clinical ABR protocol guidelines for the use of $\mathrm{BC} \mathrm{ABR}$ to characterize the degree of hearing loss and to differentiate the types (sensorineural or conductive) $[10,11]$, none of them may provide comprehensive and insight information for usable reason of BC ABR. In this review, weaimed to evaluate the characteristics and the efficacy of BC ABR for the treatment of patients clinically with hearing disorders through a systemic review, and to create a practical decision-making tool for the clinicians.

\section{Development of Bone-Conduction ABR}

In the past 40 years, several studies have evaluated the influence of the binding force to the vibrator's head on the hearing threshold of adults [12-14]. As the force on the head coupled to the vibrator increases, less energy is needed to establish an accurate threshold level due to the characteristics of the three BC pathway [i.e., distortional, inertial (ossicular), and external canal (osteotympanic)]. According to Harris, et al. [12], a slight shift in the threshold was suggested with a binding force of over $400 \mathrm{~g}$. Similarly, König [13] noted a variation in the threshold with varying bond strengths less than $750 \mathrm{~g}$. However, he observed BC threshold changes when the binding force exceeded $400 \mathrm{~g}$. In addition, Whittle [14] suggested that, while the average hearing threshold improves with an overall increasing force, the variation among subjects was quite large. These discordance from many researchers have raised concerns about the reliability of osteoconduction in
ABR records. The vibrator to head coupling method reported in previous investigations has demonstrated consistent results with $\mathrm{ABRs}$ to $\mathrm{BC}$ clicks in infants $[7,8,15]$. Currently, specific techniques and results for $\mathrm{BC}$ ABR have been well documented by the British Columbia Early Hearing Program [10] (please visit this reference).

\section{Placements for Bone Oscillator}

While conducting BC ABR tests, many clinicians have experienced large amounts of artifacts. This is true, especially at high intensities with $50 \mathrm{~dB}$ above normal hearing level (nHL) and also at earlier waves such as Wave I. To avoid stimulus artifacts, the bone oscillator should be placed high on the temporal bone, and the inverting electrode should be placed on the earlobe, mastoid, or nape of the neck. Artifacts could also be reduced with an alternating phase stimuli. Since the output of most bone oscillators is around 45 to $55 \mathrm{~dB} \mathrm{nHL}$, it becomes difficult to distinguish between sensorineural or mixed hearing losses when the losses by bone exceed this range. This output limitation of the bone oscillator is a drawback.

A previous study that examined different oscillator placements for $\mathrm{BC}$ ABR reported that the wave $\mathrm{V}$ affects the placement of the oscillators in infants, although adults were less affected by the bone vibrator. In addition, infants showed a delayed latency of wave $\mathrm{V}$ in the occipital and frontal lobe than in the temporal lobe for both $\mathrm{AC}$ and BC ABR [8]. Stuart, et al. [7] reported on BC ABR for three different placements in temporal bone and found that the posterior and supero-posterior placements could generate clearer waveforms with shortened latency than in the superior placement. De Freitas, et al. [16] reported that oscillator placement on the mastoid could estimate a lower level of thresholds than on the forehead. The density and thickness of the cranial bones and fontanelles, which comprises any soft membranous gap between the cranial bones that make up the calvaria of a fetus or an infant, are factors that can affect oscillator placement for $\mathrm{BC}$ ABR in adults and infants [7,8]. Table 1 provides useful information for determining the bone oscillator placement for clinical application.

\section{Stimuli for Bone-Conduction ABR}

Since brief click stimuli have no frequency specificity, clinicians are unaware of which frequencies particularly contribute to the click limit during testing. In this regard, tone stimulation would be useful to obtain frequency-specific thresholds. Although click ABR thresholds are known to be insufficient, we currently still use broadband click stimuli for many clinical 
Table 1. Summary of bone oscillator placement for bone-conduction auditory brainstem responses recommended by several studies

\begin{tabular}{|c|c|c|c|c|}
\hline & Yang, et al. [8] & Yang and Stuart [15] & Stuart, et al. [7] & De Freitas, et al. [16] \\
\hline \multicolumn{5}{|l|}{ Subjects } \\
\hline \multirow[t]{3}{*}{ Age (years) } & Neonates & $0-4 \mathrm{~m}$ & $38-42$ weeks & $20-30$ yearrs \\
\hline & 1-year-old infants & & & \\
\hline & Adults & & & \\
\hline Number & 10 individuals of each group & 48 & 25 & 22 \\
\hline Hearing condition & Normal & At-risk for hearing loss & Normal & Normal \\
\hline \multicolumn{5}{|l|}{ Consideration factors } \\
\hline Transducer & Radioear B-70A vibrator & $\begin{array}{l}\text { Radioear B-70B } \\
\text { vibrator }\end{array}$ & $\begin{array}{l}\text { Radioear B-70B } \\
\text { vibrator }\end{array}$ & $\begin{array}{l}\text { Radioear B-70B } \\
\text { vibrator }\end{array}$ \\
\hline Placement & Temporal bone & $\begin{array}{l}\text { Supero-posterior auricular } \\
\text { position }\end{array}$ & $\begin{array}{l}\text { Supero-posterior of } \\
\text { temporal bone }\end{array}$ & Mastoid \\
\hline
\end{tabular}

Table 2. Summary of measurement parameters for bone-conduction auditory brainstem responses recommended by several studies

\begin{tabular}{llllc}
\hline & \multicolumn{1}{c}{ Foxe and Stapells [17] } & \multicolumn{1}{c}{ Hall [9] } & Hatton, et al. [5] & De Freitas, et al. [16] \\
\hline Stimuli & Tone burst (4:2:4 and 1:0.5:1 ms plateau) & Clicks & Brief tone & Click \\
Filter (Hz) & $30-3,000$ & $30-3,000$ & $30-1,500$ & $30-3,000$ \\
Array & $C_{z}-\mathrm{Ml} / \mathrm{M} 2$ & $\mathrm{~F}_{\mathrm{z}}$-Al/A2 & Fpz-M1/M2 & $\mathrm{F}_{\mathrm{z}}$-Al/A2 \\
Polarity & Alternating & Alternating & Alternating & Alternating \\
Rate $(/ \mathrm{sec})$ & 39.1 & 7.1 & 39.1 & 27.7 \\
Sweeps & 2,000 & More than 2,000 & 2,000 & 2,000 \\
\hline
\end{tabular}

ABR thresholds. Rather, tone-evoked ABR tests have been used successfully in both research and clinical settings [1]. In particular, tone-evoked ABR for BC stimulation has been used for at least 20 years for research and clinical purposes. For example, Stapells and Ruben [6] investigated BC ABR using 500 and 2,000 Hz tones for forty-eight infants (mean age: 6.1 months) with either normal hearing (24 ears) or conductive hearing loss (41 ears). They suggested that clinically $\mathrm{nHLs}$ could be attained at ABR thresholds to BC stimuli of $30 \mathrm{~dB} \mathrm{nl}$ for $2000 \mathrm{~Hz}$ and $20 \mathrm{~dB}$ nHL for $500 \mathrm{~Hz}$.

Unfortunately, although reference equivalent threshold sound pressure levels have been provided for click and tone-pip stimuli used for AC ABR by ISO 389-6 since 2007, there is no standard reference equivalent force threshold levels for tonepip stimuli of BC ABR yet, but only for click BC ABR. Furthermore, there are few published reports on tone-evoked $\mathrm{BC}$ ABR for patients with hearing loss. Vander Werff, et al. [18] did not find meaningful thresholds of BC ABR from infants with sensorineural hearing loss due to a very small study size ( $\mathrm{n}$ is 2 for $500 \mathrm{~Hz}$ and $\mathrm{n}=9$ for 2,000 Hz). More recently, Hatton, et al. [5] successfully obtained thresholds with $98 \%$ accuracy in 500 and $2,000 \mathrm{~Hz} \mathrm{BC}$ tone stimuli to differentiate children with conductive and sensorineural hearing loss. Table 2 shows the measurement parameters for various stimuli that have clinical utility.

\section{Threshold of Bone-Conduction ABR}

By presenting stimuli to the $\mathrm{BC}$ pathway, $\mathrm{BC} \mathrm{ABR}$ is able to perceive hearing loss and neural disorders for patients with limited AC ABR or behavioral (i.e., pure-tone audiometry) testing. This characteristic of BC ABR allows it to be utilized not only for patients who have low reliable AC ABR outcomes due to diseases of the AC pathway but also infants and toddlers [3]. Moreover, there are many features of BC ABR. As mentioned earlier, while AC ABR transmits stimuli using headphones or earphones through the AC pathway, BC ABR presents stimuli with the oscillator through the temporal bone directly to the cochlea [8]. The vibration of the temporal bone and cochlea generates binaural attenuation caused by signal dispersion in the cranial bone.

This difference between $\mathrm{AC}$ and $\mathrm{BC}$ ABR manifests in the latency of wave $\mathrm{V}$. The wave $\mathrm{V}$ latency of $\mathrm{BC}$ ABR was approximately $0.5 \mathrm{~ms}$ longer with a larger amplitude than $\mathrm{AC}$ ABR [19]. The amplitude of wave V and early waveform modalities seem to change with the level of the stimuli. For high level stimuli, BC ABR was not differentiated from AC ABR for the amplitude of wave $\mathrm{V}$ and early waveforms. In contrast, with low level stimuli (under $55 \mathrm{~dB} \mathrm{nHL}$ ), the latency of wave $\mathrm{V}$ was delayed approximately $0.5 \mathrm{~ms}$ compared to AC ABR, and the early waveforms were not clearly distinguished. A previous study that compared both infant and adult $\mathrm{AC}$ and $\mathrm{BC}$ 
ABRs reported that infants showed similar thresholds for both $\mathrm{ABR}$ tests (3.75 and $1.25 \mathrm{~dB} \mathrm{nHL}$, respectively), whereas adults showed a significant difference between $\mathrm{AC}$ and $\mathrm{BC}$ ABR thresholds (3.75 and $18.75 \mathrm{~dB} \mathrm{nHL}$, respectively).

\section{Latency of Bone-Conduction ABR}

BC ABR waves typically appear more rounded than more conventional AC ABR. The maximum output of $50 \mathrm{~dB} \mathrm{nHL}$ stimulated by the bone oscillator should appear similar to the $50 \mathrm{~dB}$ nHL response of AC for people with normal hearing or with a mild sensorineural hearing loss. If a patient has conductive hearing loss, the latencies for AC are shifted when compared to the latencies of BC ABR.

\section{Comparison with AC}

Several studies have found that the latencies of the ABR waves to BC stimuli are approximately 0.16 to $0.88 \mathrm{~ms}$ longer than those to AC stimuli $[8,19]$. Such a latency discrepancy has been thought to be due to the low pass filter characteristics of the bone vibrator. In other words, $\mathrm{BC}$ stimuli would excite more apical cochlear regions with a longer traveling wave delay than the corresponding AC stimuli $[8,19]$. Durrant and Hyre [20] had suggested that this delayed $\mathrm{BC}$ response may be related to the propagation delay and low pass filtering involved in skull vibration. Gorga, et al. [21] have also explained that there may be some inherent differences in the transmission of energy between AC and BC stimuli.

\section{Age effect}

As stated previously, the wave $\mathrm{V}$ latencies derived from $\mathrm{BC}$ $\mathrm{ABR}$ are approximately $0.5 \mathrm{mms}$ longer than those from a same intensity of AC stimulus. For infants, the wave V latencies evoked by $\mathrm{BC}$ clicks are shorter than those of AC clicks [8]. Many researchers have insisted that these differences can be explained by the changes of skull due to aging.

\section{Gender effect}

In general, the studies documenting gender differences in the AC ABR have reported shorter absolute wave latencies and larger wave amplitudes for female participants. To examine the gender differences of infants, Stuart and Yang [22] measured wave $\mathrm{V}$ latency thresholds of two hundred and two full-term neonates (103 males and 99 female) using AC and BC click stimuli of 30,45 , and $60 \mathrm{~dB}$ nHL and 15 and $30 \mathrm{~dB}$ $\mathrm{nHL}$, respectively. The results demonstrated that female newborns showed significant shorter wave $\mathrm{V}$ latencies than male newborns for AC click stimuli (i.e., approximately $0.2-0.3$ $\mathrm{ms}$ ). However, there was no significant gender difference in wave $\mathrm{V}$ latencies when applying click stimuli for BC ABR.

\section{Clinical Outcomes}

\section{Maturation: infants and young children with normal hearing to adults \\ BC ABR can estimate cochlear function in infants and help} identify any types of hearing loss that could be present $[6,8$, 15,23]. Hooks and Weber [23] studied BC ABR stimulated by clicks in premature infants and showed that there were large differences in ABR results between infants and adults in terms of morphology, latency, and amplitude. These differences are thought to be caused by the maturation of the auditory pathway in infant. The morphology, latency, and amplitude of infants' ABR results showed similar characteristics as adults. Full-term infants showed the wave I, III, V, and a delayed interwave and absolute latency compared to adults [24]. Their latency of wave I becomes adult-like by six to twenty-four weeks, and waves III and V become adult-like by 18 months of chronological age [24]. BC ABR showed more delayed latency than $\mathrm{AC} A B R$, in general.

BC ABR appears to be an incomplete technique for the screening of infant conductive hearing losses in the intensive care nursery; huge stimulus artifacts are generated which can consequently increase the bone vibrator to higher intensities. This can obscure the first 1 to $2 \mathrm{~ms}$ of the ABR waveforms. Another reason is the subject variation of the transmission of vibrational stimuli, which seems to be due to individual differences in skull impedance. However, Yang, et al. [8] showed that proper vibrator placements at the temporal bone could make BC ABR a feasible and reliable diagnostic tool for testing infants while masking ipsilateral AC pathway. Stuart, et al [7] suggested that the placement of bone vibrators around postauricular areas may affect $\mathrm{ABR}$ recordings in newborn infants. He also investigated ABR thresholds to $\mathrm{AC}$ and $\mathrm{BC}$ clicks in 40 full-term neonates [25]. This study suggested that a dissimilarity exists between $\mathrm{ABR}$ thresholds to AC clicks but not to BC clicks in neonates less than 48 hours old and those between 49 and 96 hours. Cone-Wesson and Ramirez [26] examined the ABR threshold difference in gender for ABR BC clicks and tone bursts at 500 and 4,000 Hz for 60 newborns. In another study by Stuart and Yang [22], gender differences were not observed in latency or threshold for AC ABR compared to BC ABR stimulated by clicks.

\section{Pathology: congenital EACA}

As mentioned previously, infants showing elevated ABR thresholds to AC stimuli should be further tested with BC stimuli. Infants with atresia, microtia, otitis media, and other 
outer/middle ear abnormalities, as well as sensorineural hearing losses, should require BC ABR testing. Furthermore, infants who have had a considerable amount of amniotic fluid in their middle ear space may need to be tested with BC ABR while considering this fluid usually disappears by 48 hours after birth. Five paths contributing to BC hearing the clinicians should consider are as followed: 1) external auditory canal sound radiation; 2) inertia of the ossicular chain; 3) inertia of the cochlear fluid; 4) compression of cochlear walls; and 5) pressure transmission from the cerebrospinal fluid [27].

Patients with congenital hearing aural atresia predominantly exhibit a lack of inertia in the ear chains. For example, Chen, et al. [4] compared the differences in AC and BC ABR latency for children with congenital EACA and normal hearing. Although the EACA group demonstrated significantly higher (i.e., worse) AC ABR thresholds due to the pathology in the $A C$ pathway, there were no significant differences in $\mathrm{BC}$ $\mathrm{ABR}$ and $\mathrm{BC} \mathrm{ABR}$ latency between groups. These findings demonstrate that $\mathrm{BC} \mathrm{ABR}$ had clinical utility for the examination of cochlear, auditory nerve, and central auditory pathway function. Table 3 provides the recommended absolute latencies of waves I, III, and V conducted by BC ABR for both infants and adults who structurally and functionally had normal hearing, and infants with atresia. The absolute latencies will be helpful for utilizing $\mathrm{BC} \mathrm{ABR}$ in the clinical setting.

\section{Bone-Conduction ABR Applications for Bone-Conduction Hearing Implants}

In case of clinical purpose, ABR assessed to estimate thresholds and to classify types of hearing losses. The Joint Committee on Infant Hearing (2007), National Institutes of Health

Table 3. Recommended absolute latencies of waves I, III, and V conducted by bone-conduction auditory brainstem response for specific populations

\begin{tabular}{|c|c|c|c|}
\hline Population & Wave I & Wave III & Wave V \\
\hline \multicolumn{4}{|l|}{ Norm } \\
\hline Infants $(1-20$ & $2.19 \pm 0.45$ & $4.83 \pm 0.51$ & $6.65 \pm 0.33[4]$ \\
\hline \multirow[t]{7}{*}{ months) } & & & $7.24 \pm 0.36[23]$ \\
\hline & & & $8.38 \pm 0.36$ [9] \\
\hline & & & $8.41 \pm 0.44[33]$ \\
\hline & & & $8.37 \pm 0.54[33]$ \\
\hline & & & $11.05 \pm 0.84[21]$ \\
\hline & & & $9.89 \pm 0.87[21]$ \\
\hline & & & $8.63 \pm 0.32[37]$ \\
\hline \multirow[t]{2}{*}{ Adults } & & & $6.59 \pm 0.36[23]$ \\
\hline & & & $6.79 \pm 0.41[19]$ \\
\hline \multicolumn{4}{|l|}{ Pathology } \\
\hline Atresia & $2.27 \pm 0.25$ & $4.99 \pm 0.44$ & $6.78 \pm 0.38[4]$ \\
\hline
\end{tabular}

Unit: milliseconds (ms)
(1993) and American Speech-Language-Hearing Association (2004) all support the use of ABR for universal newborn hearing screening. BC ABR can be used to specifically identify the different types of hearing losses. However, the patients usually limit for infants and not apply routinely. Importantly, $\mathrm{BC} A B R$ could be an alternative to $\mathrm{AC} A B R$ in patients with diseases of the AC pathway. BC ABR showed larger amplitudes of wave $\mathrm{V}$ and clearer waveforms for patients with atresia or microtia. These advantages of BC ABR could be applied for bone anchored hearing aid (BAHA) utility and offer definite testing results. In conclusion, overall comparison and understanding of $\mathrm{BC}$ pure-tone audiometry, $\mathrm{BC} \mathrm{ABR}$, and results by oscillators using BAHA may lead to scientific evidence for mapping BAHA and expect accurate performance of BAHA users.

Recently, BAHAs have been placed in patients with conductive hearing loss or congenital atresia. In 2001, Setou, et al. [28] revealed the existence of a BC-binaural interaction component (BIC) in normal hearing adults and suggested that the bilateral fitting of $\mathrm{BC}$ hearing aids in children with atresia allow for the localization of the sound. Since then, many studies have also reported that BC-BIC is present in children with congenital atresia as well as normal-hearing adults, and the gross response properties of BIC are similar between these two populations. Such a BAHA system has several clinical applications and has proved to be reliable. In some cases, it is necessary to estimate aided and unaided pure-tone audiograms by objective methods, especially for passively cooperative subjects like young children; to this end, Eggermont has developed a stimulated one. Electroencephalography receives typical changes with time correlation and sound stimulation [17]. ABR appears as a series of vertex positive waves occurring within 15 ms of the start of transient stimulation. An auditory evoked potential (AEP) measurement using BAHA has been reported for patients with unilateral sensorineural hearing loss [29]. A free field sound stimulus with a sound intensity level of $80 \mathrm{~dB}$ $\mathrm{HL}$, requires masking or occlusion of healthy ears, was used in this study. According to Rahne, et al. [30], recording ABR and AEP by delivering a stimulus via BAHA yields a response waveform comparable to the response waveform induced by earphone stimulation. They suggested that the thresholds of ABR and AEP seem to match the subject's normal hearing.

\section{Conclusion}

In the clinical setting, ABR audiometry has been used as a powerful objective tool to evaluate hearing sensitivity and to diagnose various types of hearing loss and neurological disorders. Although BC ABR could be an alternative to AC ABR 
as it overcomes some limitations of the latter, the majority of clinicians rarely use it due to a lack of knowledge and no routine test administration. Based on several clinical BC ABR protocol guidelines mentioned in this review, a comparison between $\mathrm{AC}$ and $\mathrm{BC} \mathrm{ABR}$ should be applied to characterize the degree of hearing loss and to differentiate its types. Moreover, BC ABR can be helpful in determining subsequent testing, and can be important for both appropriate follow up and parent counselling.

\section{Acknowledgments}

This work was supported by the Ministry of Education of the Republic of Korea and the National Research Foundation of Korea (NRF2015S1A3A2046760) and by 'Industrial Core Technology Development Program of Biomedical Devices' funded by the Ministry of Trade, Industry \& Energy (MI, Korea) (\#10051518).

\section{Conflicts of interest}

The authors have no financial conflicts of interest.

\section{REFERENCES}

1) Stapells DR, Oates P. Estimation of the pure-tone audiogram by the auditory brainstem response: a review. Audiol Neurootol 1997;2:25780.

2) Gorga MP, Johnson TA, Kaminski JR, Beauchaine KL, Garner CA, Neely ST. Using a combination of click- and tone burst-evoked auditory brain stem response measurements to estimate pure-tone thresholds. Ear Hear 2006;27:60-74.

3) Small SA, Stapells DR. Normal brief-tone bone-conduction behavioral thresholds using the B-71 transducer: three occlusion conditions. J Am Acad Audiol 2003;14:556-62.

4) Chen K, Jiang C, Wu Q, Sun Y, Shi R. A new flap technique for reconstruction of microtia and congenital aural atresia. Indian J Surg 2015;77(Suppl 3):1237-41.

5) Hatton JL, Janssen RM, Stapells DR. Auditory brainstem responses to bone-conducted brief tones in young children with conductive or sensorineural hearing loss. Int J Otolaryngol 2012;2012:6.

6) Stapells DR, Ruben RJ. Auditory brain stem responses to bone-conducted tones in infants. Ann Otol Rhinol Laryngol 1989;98(12 Pt 1):941-9.

7) Stuart A, Yang EY, Stenstrom R. Effect of temporal area bone vibrator placement on auditory brain stem response in newborn infants. Ear Hear 1990;11:363-9.

8) Yang EY, Rupert AL, Moushegian G. A developmental study of bone conduction auditory brain stem response in infants. Ear Hear 1987;8:244-51.

9) Hall III JW. Bone-conduction ABR: clinically feasible and clinically valuable. Hear J 1994;47:10,35-7.

10) British Columbia Early Hearing Program (BCEHP). BCEHP Audiology Assessment Protocol, 2012 [cited 2015 Jan 8]. Available from: https://www.researchgate.net/publication/242482099.

11) American Speech-Language-Hearing Association. Guidelines for the audiologic assessment of children from birth to 5 years of age, 2014 [cited 2014]. Available from: http://www.asha.org/policy.

12) Harris JD, Haines HL, Myers CK. A helmet-held bone conduction vibrator. Laryngoscope 1953;63:998-1007.

13) König E. Variations in bone conduction as related to the force of pressure exerted on the vibrator (Translations of the Beltone Institute for Hearing Research, 6). Chicago, IL: Beltone Institute for Hearing Research;1957.

14) Whittle LS. A determination of the normal threshold of hearing by bone conduction. J Sound Vib 1965;2:227-48.

15) Yang EY, Stuart A. A method of auditory brainstem response testing of infants using bone-conducted clicks. 1SLPA/ROA 1990;14:69-76.

16) de Freitas VS, de Alvarenga KF, Morettin M, de Souza EF, Filho $\mathrm{OA}$. [Bone conduction auditory brainstem responses in normal hearing individuals]. Pro Fono 2006;18:323-30.

17) Foxe JJ, Stapells DR. Normal infant and adult auditory brainstem responses to bone-conducted tones. Audiology 1993;32:95-109.

18) Vander Werff KR, Prieve BA, Georgantas LM. Infant air and bone conduction tone burst auditory brain stem responses for classification of hearing loss and the relationship to behavioral thresholds. Ear Hear 2009;30:350-68.

19) Cornacchia L, Martini A, Morra B. Air and bone conduction brain stem responses in adults and infants. Audiology 1983;22:430-7.

20) Durrant JD, Hyre R. Observations on temporal aspects of bone-conduction clicks: real head measurements. J Am Acad Audiol 1993;4: 213-9.

21) Gorga MP, Kaminski JR, Beauchaine KL, Bergman BM. A comparison of auditory brain stem response thresholds and latencies elicited by air- and bone-conducted stimuli. Ear Hear 1993;14:85-94.

22) Stuart A, Yang EY. Gender effects in auditory brainstem responses to air- and bone-conducted clicks in neonates. J Commun Disord 2001; 34:229-39.

23) Hooks RG, Weber BA. Auditory brain stem responses of premature infants to bone-conducted stimuli: a feasibility study. Ear Hear 1984; 5:42-6.

24) Salamy A, McKean CM, Buda FB. Maturational changes in auditory transmission as reflected in human brain stem potentials. Brain Res 1975;96:361-6.

25) Stuart A, Yang EY, Green WB. Neonatal auditory brainstem response thresholds to air- and bone-conducted clicks: 0 to 96 hours postpartum. J Am Acad Audiol 1994;5:163-72.

26) Cone-Wesson B, Ramirez GM. Hearing sensitivity in newborns estimated from ABRs to bone-conducted sounds. J Am Acad Audiol 1997;8:299-307.

27) Zhang L, Gao N, Yin Y, Yang L, Xie Y, Chen Y, et al. Bone conduction hearing in congenital aural atresia. Eur Arch Otorhinolaryngol 2016;273:1697-703.

28) Setou M, Kurauchi T, Tsuzuku T, Kaga K. Binaural interaction of bone-conducted auditory brainstem responses. Acta Otolaryngol 2001;121:486-9.

29) Tringali S, Marzin A, Dubreuil C, Ferber-Viart C. Bone-anchored hearing aid in unilateral inner ear deafness: electrophysiological results in patients following vestibular schwannoma removal. Acta Otolaryngol 2008;128:1203-10.

30) Rahne T, Ehelebe T, Rasinski C, Götze G. Auditory brainstem and cortical potentials following bone-anchored hearing aid stimulation. J Neurosci Methods 2010;193:300-6. 